\title{
A challenging hernia: primary venous aneurysm of the proximal saphenous vein
}

\author{
Ch. Tschuor $\cdot$ D. Dindo $\cdot$ P.-A. Clavien $\cdot$ D. Hahnloser
}

Received: 25 August 2011 / Accepted: 7 February 2012 / Published online: 17 March 2012

(C) Springer-Verlag 2012

\begin{abstract}
Introduction Primary venous aneurysm is a rare, but essential consideration in the differential diagnosis of an inguinal and femoral hernia.

Methods We report a case of a 43-year-old man who was referred for evaluation and treatment of a femoral hernia.

Results The patient presented with a 3-month history of an asymptomatic tumor on his right upper inner thigh. Physical examination noted a non-tender, non-indurated tumor.

Conclusion Surgical exploration demonstrated a primary venous aneurysm of the proximal saphenous vein.
\end{abstract}

Keywords Saphenous vein - Primary venous aneurysm . Hernia

\section{Introduction}

Primary venous aneurysms of the proximal saphenous vein are uncommon [1]. The aneurysms are usually congenital or develop from defective venous tissue [2]. The clinical presentation is usually an asymptomatic, superficial mass misdiagnosed and confused with an inguinal or femoral

Ch. Tschuor $\cdot$ D. Dindo $\cdot$ P.-A. Clavien $\cdot$ D. Hahnloser Department of Visceral and Transplantation Surgery, University Hospital of Zurich, Raemistrasse 100, 8091 Zurich, Switzerland

D. Dindo

Department of Surgery, Triemli Hospital,

Birmensdorferstrasse 497, 8063 Zurich, Switzerland

D. Hahnloser ( $\square)$

Department of Visceral Surgery, University Hospital (CHUV),

Rue du Bugnon 46, 1011 Lausanne, Switzerland

e-mail: Dieter.Hahnloser@chuv.ch hernia [3]. Since misdiagnosis can lead to severe morbidity, we intend to capture the sense for this clinical image. We report a case of a primary venous aneurysm of the proximal saphenous vein.

\section{Case report}

A 43-year-old man presented with a 3-month history of an asymptomatic tumor on his right inner thigh to our outpatient clinic (Fig. 1). The tumor was present when standing; however, it disappeared in the supine position. Physical examination showed a non-tender, non-indurated, slightly relocatable tumor with a positive cough impulse. The patient's medical history was notable for HIV first diagnosed in 1998 with general lymphadenopathy (A2 CDC). He has been treated several times for extensive endo- and perianal Condylomata accuminata with cryo- and lasertherapy as well as with Aldara (Imiquimod) in our clinic.

Further diagnostics were indicated. An MRI of the pelvis and the thighs showed no evidence for a femoral or an inguinal hernia, nor did it show any other pathological findings such as lymphadenopathy or vascular malformations. Further diagnostic workup would have included a Doppler ultrasound in the supine and standing position. However, since surgical treatment for the Condylomata accuminata under general anesthesia had not yet been planned, we decided to perform a surgical exploration of this tumor. Surgical exploration showed a venous aneurysm of the proximal saphenous vein (Fig. 2). We performed tangential excision with lateral venorrhaphy. Histologic examination confirmed a primary venous aneurysm. The postoperative course was uneventful. At 4-month follow-up, the patient appeared healthy and no recurrence of the primary venous aneurysm was found. 


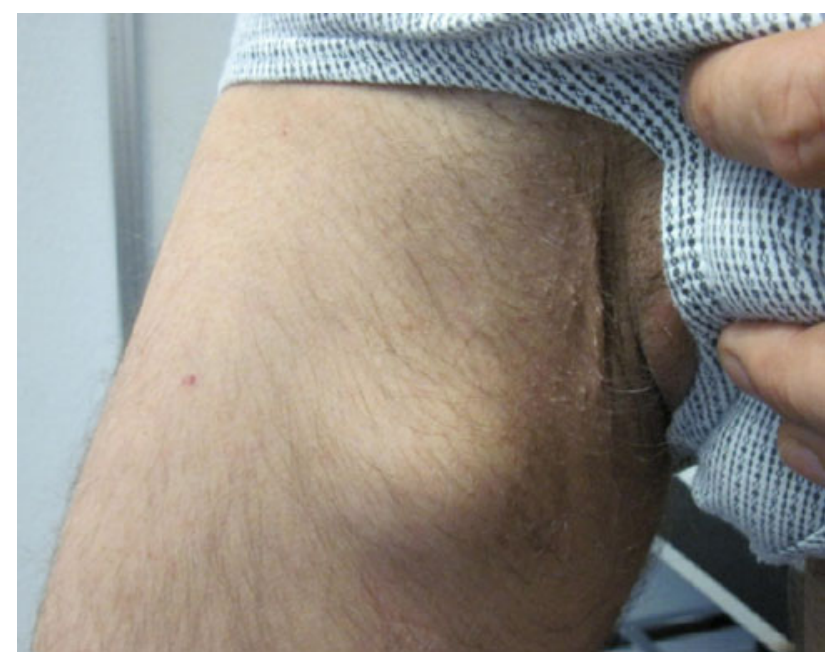

Fig. 1 The clinical presentation of the asymptomatic tumor on the right upper inner thigh

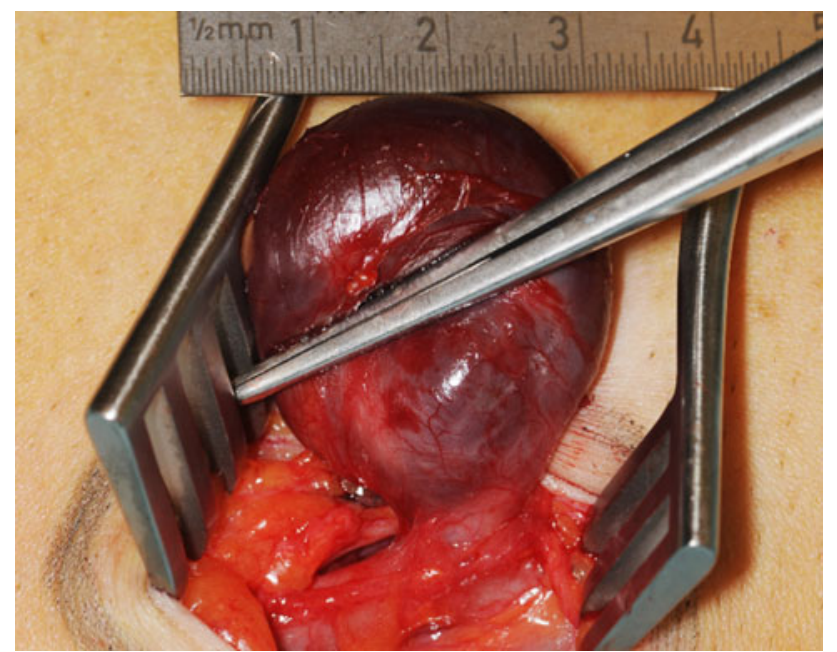

Fig. 2 Intraoperative findings of the primary venous aneurysm of the proximal saphenous vein

\section{Discussion}

Concerning the location of the tumor and the clinical findings in the physical examination, three main diagnoses should be considered: First an inguinal or rather a femoral hernia could cause these clinical findings. Second, a possible lymphadenopathy in this patient suffering from HIV should be kept in mind. Also, a lipoma or another subcutaneous tumor could be possible. However, the clinical presentation would be rather atypical, since they would not disappear in the supine position. Third, even though it is a very rare cause, the clinical findings could be due to a vascular arterial or venous malformation. The manifestation of a venous disorder such as a varicose of the proximal great saphenous vein has to be considered since it presents with similar clinical signs $[4,5]$.

Osler was the first to recognize venous aneurysms as a clinical entity in 1913 [6]. A venous aneurysm is a rare, localized saccular or fusiform dilatation of a venous segment with a caliber at least $50 \%$ greater than the normal trunk [4]. It may be the source of pulmonary embolism and can result in death [7]. A primary venous aneurysm is defined as an area of venous dilatation in communication with a main venous structure by a single channel. It presents all three layers of the normal vein. Primary venous aneurysms of the proximal saphenous vein are uncommon [1] and were first described by May and Nissel [7]. There are cases in literature in all ages with equal distribution between sexes [8]. The aneurysms are usually congential or develop from defective venous tissue [2]. Secondary or acquired venous aneurysms are usually found in adults and are associated with inflammation, trauma or stretch injury $[9,10]$. The clinical presentation is usually an asymptomatic, superficial mass misdiagnosed as an inguinal hernia [3]. In rare cases, the clinical presentation is a deep venous thrombosis or a pulmonary embolism. The diagnosis of the primary venous aneurysm is usually achieved by a Doppler ultrasound. We missed the diagnosis, since the MRI is performed in supine position. Therefore, the aneurysm was not filled, and no aneurysmal dilatation was detectable. A surgical management is to be preferred because of its high risk of thromboembolism [10]. The surgical approach is defined by the location of the aneurysm. Surgical options are ligation, resection with end to end anastomosis, resection with vein graft and aneurysmorrhaphy or tangential excision with lateral venorraphy [8].

\section{Conclusion}

In comparison with hernias or lymphadenopathy in the inguinal region, primary venous aneurysms are extremely rare. They are most frequently seen in patients referred for evaluation and treatment of an inguinal or femoral hernia and seldom considered in the differential diagnosis. Yet, the awareness of this entity is important since misdiagnosis can lead to severe morbidity and mortality.

\section{References}

1. Uematsu M, Okada M (1999) Primary venous aneurysms: case reports. Angiology 50:239-244

2. Majeski J (2002) Surgical repair of primary saphenous vein aneurysm of the proximal leg after initial presentation as an inguinal hernia. Am Surg 68:999-1002

3. Paes T, Andrews S, Wyatt A (1991) Acquired venous aneurysms. Br J Sports Med 25:149-150 
4. Eklof B, Perrin M, Delis KT, Rutherford BR, Gloviczki P (2009) Updated terminology of chronic venous disorders: the VEINTERM transatlantic interdisciplinary consensus document. J Vasc Surg 49:498-501

5. Labropoulos N, Kokkosis AA, Spentzouris G, Gasparis AP, Tassiopoulos AK (2010) The distribution and significance of varicosities in the saphenous trunks. J Vasc Surg 51:96-103

6. Osler W (1913) An arteriovenous aneurysm of the axillary vessels of thirty years duration. Lancet 2:1248

7. May R, Nissel R (1968) Aneurysma der Vena Poplitea. Rofo Fortschr Geb Rontgenstr Neuen Bildgeb Verfahr 180:402-403
8. Marcucci G, Accrocca F, Antignani PL, Siani A (2010) An isolated aneurysm of the thigh anterolateral branch of the greater saphenous vein in a young patient presenting as an inguinal hernia. Interact Cardiovasc Thorac Surg 10:654-655

9. Gillespie DL, Villavicencio JL, Gallagher C, Chang A, Hamelink JK, Fiala LA, O’Donnell SD, Jackson MR, Pikoulis E, Rich NM (1997) Presentation and management of venous aneurysms. J Vasc Surg 26:845-852

10. Schatz IJ, Fine G (1962) Venous aneurysms. N Engl J Med 266:1310-1312 